\title{
Health effects of $\mathrm{PM}_{2.5}$ constituents and source contributions in major metropolitan cities, South Korea
}

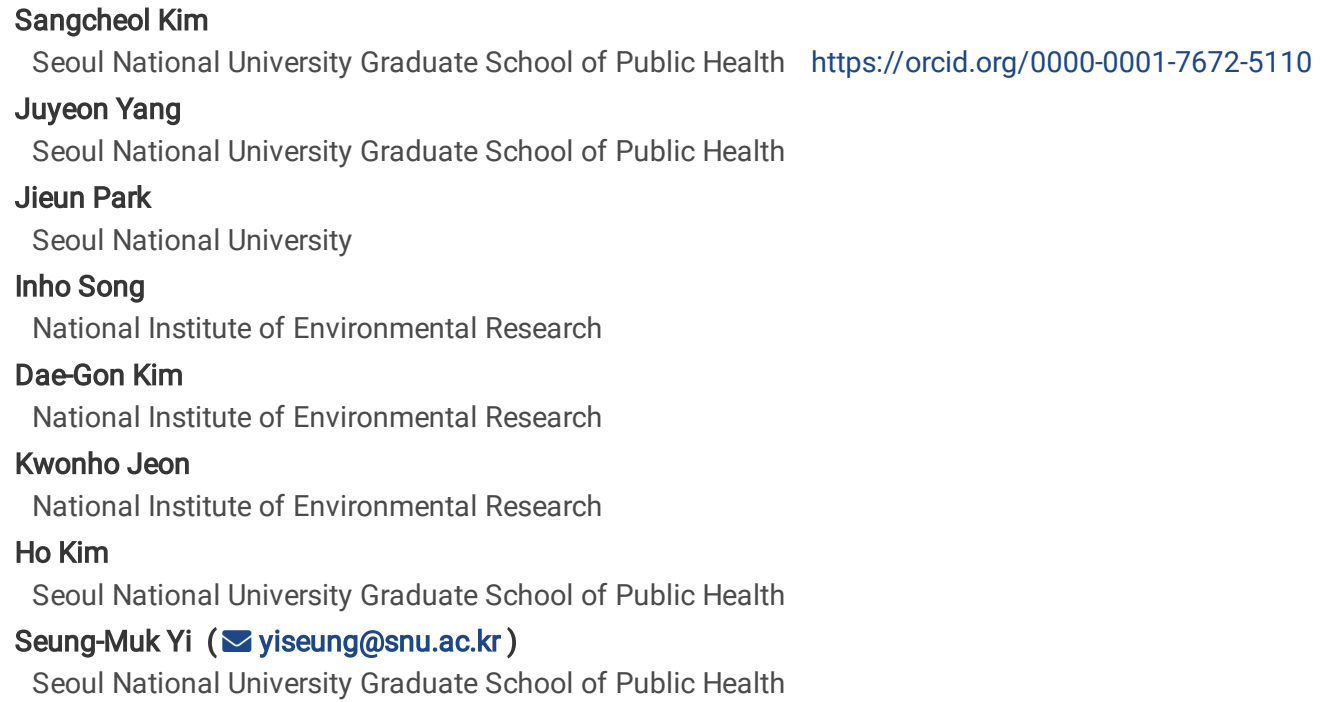

\section{Research Article}

Keywords: PM2.5, health effects, mortality, generalized linear model, PMF, South Korea

Posted Date: October 25th, 2021

DOI: https://doi.org/10.21203/rs.3.rs-996785/v1

License: () (1) This work is licensed under a Creative Commons Attribution 4.0 International License. Read Full License 


\section{Abstract}

South Korea is one of the East Asian countries suffering severe ambient $\mathrm{PM}_{2.5}$ pollution which has been continuously reported to a risk factor driving both death and disability in many parts of the world. We investigated the associations of cause-specific mortality with both $\mathrm{PM}_{2.5}$ chemical constituents and source contributions in four metropolitan cities, namely Seoul, Daejeon, Gwangju and Ulsan, by applying the generalized linear model (GLM) to the results of the positive matrix factorization (PMF) modeling. The cities represent each of four air control zone designated as of April 2020 by the special law for controlling air pollution on a regional basis in South Korea. We found that that the interquartile range (IQR) increase in the concentration $\mathrm{PM} \mathrm{M}_{2.5}$ chemical constituents largely increased the relative risk (RR) of mortality, whereas the significance and strength of the associations were different among the cities. In addition, the effects of unit increase in source contributions also differed depending on regions. In conclusion, the results of this study suggest the $\mathrm{PM}_{2.5}$ compositional characteristics may have influenced the heterogeneous health of $\mathrm{PM}_{2.5}$ exposure among the cities. Therefore, the plans and policies based on the regional characteristics of $\mathrm{PM}_{2.5}$ composition and health risk need to be implemented along with the reduction of total mass concentration of $\mathrm{PM}_{2.5}$ for mitigating adverse health effects efficiently from a perspective of public health.

\section{Introduction}

Ambient particulate matters have become a one of the most concerning factors affecting human health in numerous large cities around the globe. Especially $\mathrm{PM}_{2.5}$, defined as particle with aerodynamic diameter less than or equal to $2.5 \mathrm{\bigotimes}$, has been reported to have more severe impacts on human body than bigger particles due to physiochemical properties (Schins et al. 2004).

First, extremely small sizes lead $\mathrm{PM}_{2.5}$ to both high mobility and difficulty of removal once inhaled into human body through breath. Unlike bigger airborne particulate matters, removed by mucociliary clearance, $\mathrm{PM}_{2.5}$ can reach the deeper region of respiratory tract and retain in the lungs for longer periods of time. Thus $\mathrm{PM}_{2.5}$ account for considerable proportions of particles observed in human pulmonary parenchyma and it is very difficult to be removed (Churg and Brauer 1997). Large surface area is another physical property of $\mathrm{PM}_{2.5}$ explaining the relationship between the exposure to $\mathrm{PM}_{2.5}$ and various health outcomes. The great specific surface area facilitates $\mathrm{PM}_{2.5}$ to act as a carrier of highly toxic compounds such as polycyclic aromatic hydrocarbons (PAHs) and transition metals (Kong et al. 2010; Pandey et al. 2013). Furthermore, the chemical heterogeneity of PM 2.5 composition also plays an important role in affecting human health because each constituent of $\mathrm{PM}_{2.5}$ has its own mechanisms and effects in terms of toxicity.

These properties of $\mathrm{PM}_{2.5}$ mostly originate from the formation processes, especially secondary formation among various gaseous precursors in the atmosphere (Wang-Li 2015). Due to the complexity of formation processes among precursors, $\mathrm{PM}_{2.5}$ is composed of various constituents including ionic compounds (e.g., sulfate, nitrate, ammonium), carbonaceous compounds (e.g., organic carbon, element carbon), trace elements (e.g., nicker, cadmium, arsenic) and other substances such as PAHs and volatile organic compounds (Ye et al. 2003; Dai et al. 2013; Amil et al. 2016). In addition, the proportions of each constituent vary considerably according to spatial and temporal conditions of the samples because $\mathrm{PM}_{2.5}$ is highly sourcedependent secondary aerosols (Cheng et al. 2012; He et al. 2012; Li et al. 2017).

In the similar context, the toxicity of $\mathrm{PM}_{2.5}$ may differ spatiotemporally and it should be evaluated exactly considering chemical composition in the region of interest for developing relevant plans or policies to reduce health effects of $\mathrm{PM}_{2.5}$. Nevertheless, many of administrative plans with regard to $\mathrm{PM}_{2.5}$ have been mostly focused on the total mass of $\mathrm{PM}_{2.5}$. The aims of this study are to investigate the effects of short-term exposure to $\mathrm{PM} 2.5$ constituents on cause-specific mortality and find the association of source contributions with mortality in four major metropolitan cities, South Korea for controlling $\mathrm{PM}_{2.5}$ from a perspective of public health.

\section{Materials And Methods}

\section{Study population}

As of April 2020, Special act on the improvement of air quality in air control zone became effective to manage the air quality efficiently and systemically on a regional basis in South Korea. According to the law, four air control zone of which air pollution is much severer than other region were designated for the country, namely Seoul metropolitan area, Middle area, Southern area, Southeastern area. The study populations were all residents in the four metropolitan cities during 2014-2018 shown in Figure 1 located in each air control zone.

Seoul (hereinafter SL) representing Seoul metropolitan area is located in the northwestern region of and is adjacent to Yellow Sea lying between mainland China on the west and north and the Korean peninsula on the east. Its area and population are $605 \mathrm{~km}^{2}$ and around 9.6 million. Daejeon (hereinafter DJ), the largest city in Middle area, is located in the central region of the country and it had a population of 1.5 million in 2019 . Gwangju (hereinafter $\mathrm{GJ}$ ) of Southern area is a city in the southwest of the country and its population and area were approximately 1.5 million and $501 \mathrm{~km}^{2}$ similar to those of Daejeon. Ulsan (hereinafter US), located in southeast part of the country and bordering the sea, had a population of 1.1 million in 2019 with total area of $1062 \mathrm{~km}^{2}$. US where two huge industrial complexes exist is widely known as a city of heavy industry (Kim et al. 2018).

\section{Air pollution and meteorological data}


National Institute of Environmental Research (NIER) under the ministry of environment, South Korea has been operating several air pollution intensive monitoring stations (APIMS) across the country to for $\mathrm{PM}_{2.5}$ speciation along with monitoring gaseous pollutants including nitrogen dioxide, sulfur dioxide. Ambient air samples are collected every day at APIMS for $24 \mathrm{~h}$ period and analyzed for chemical constituents. We obtained the measured values of $\mathrm{PM}_{2.5}$ speciation during $2014-2018$ at SL $\left(37.61^{\circ}, 126.93^{\circ}\right)$, DJ $\left(36.32^{\circ}, 127.41^{\circ}\right)$, GJ $\left(35.23^{\circ}, 126.85^{\circ}\right)$ and $\mathrm{US}\left(35.58^{\circ}, 129.32^{\circ}\right)$ sites from NIER. Chemical constituents were water-soluble ions (e.g., sulfate $\left(\mathrm{SO}_{4}{ }^{2-}\right)$, nitrate $\left(\mathrm{NO}_{3}{ }^{-}\right)$, ammonium $\left(\mathrm{NH}_{4}{ }^{+}\right)$), carbonaceous compounds (i.e., organic carbon (OC), elemental carbon (EC)) and trace elements (e.g., titanium (Ti), vanadium (V), nickel (Ni)).

Meteorological parameters are also so important factors affecting human health including morbidity and mortality that they should be included in the health effect model for control (Allen and Sheridan, 2014; Wang et al. 2019). Thus, we collected daily average temperature, relative humidity (RH) and barometric pressure (BP) data during 2014-2018 via Weather Data Service (https://data.kma.go.kr) operated by Korea Meteorological Administration.

\section{Health outcomes data}

Various health related parameters including mortality, hospital emergency room visits, out-of-hospital cardiac arrest (OHCA) have been widely used to estimate the adverse effects of exposure to $\mathrm{PM}_{2.5}$ on health in various epidemiological studies (Atkinson et al. 2014; Qiao et al. 2014; Pradeau et al. 2015). In the present study, we selected cause-specific daily mortality data as health outcomes. Daily death counts in the four cities were obtained from MicroData Integrated Service (https://mdis.kostat.go.kr) operated by the Statistics Korea. We included death counts for residents in each city as mortality data after classifying them into all-cause (non-accidental, NA, A00-R99), cardiovascular disease (CVD, I00-199) and respiratory disease (RD, J00-J99) mortality according to $10^{\text {th }}$ version of International Classification of Disease (ICD-10). This study was approved by the Seoul National University Institutional Review Board (IRB No: E2101/003-001).

\section{PMF modeling}

Among various receptor models based on chemical analyses, positive matrix factorization (PMF) and chemical mass balance (CMB) have been frequently performed for identifying the sources and estimating their contributions. Many of previous studies showed the results based on both PMF and CMB were quite comparable (Begum et al. 2007; Ke et al. 2008; Teixeira et al. 2015). But in terms of applicability, PMF is more flexible than CMB because PMF doesn't need source profiles for model execution. It is difficult to obtain suitable source profiles established for the region of interest although it strongly affects the source apportionment results. Therefore, we selected PMF to investigate the sources and their contribution for $\mathrm{PM}_{2.5}$ in the study cities. Principles and more details of PMF can be found elsewhere (Song et al. 2006; Watson et al. 2008; Manousakas et al. 2017).

As for PMF input data, we constructed the concentrations and their corresponding uncertainty data for the chemical species according to the method described in the guidebook of US EPA PMF 5.0 (Norris et al. 2008). Data values below the method detection limit (MDL) were replaced with half of the MDL and 5/6 $\times$ MDL were used as their corresponding uncertainties. For the measured values greater than the MDL, the uncertainties were calculated by the following Eq (1) and we used $10 \%$ as error fraction of each $\mathrm{PM}_{2.5}$ constituents.

Uncertainty $=\left[(\text { Error Fraction } \times \text { Concentration })^{2}+(0.5 \times \mathrm{MDL})^{2}\right]^{1 / 2}(1)$

Whole datasets on days when the mass balance and the ion balance was not satisfactory were excluded from the input data. After obtaining best solutions we also conducted error estimation using displacement method (DISP) for acquiring uncertainty estimates for each factor of the solutions (Paatero et al. 2014).

\section{Health effects analyses}

Health effects of exposure to $\mathrm{PM}_{2.5}$ can be expressed as the strength of the associations between $\mathrm{PM}_{2.5}$ constituents and health outcomes via timeseries analyses using various statistical models. Previous studies have revealed the effects of $\mathrm{PM}_{2.5}$ on health-related outcomes including mortality, morbidity using generalized linear model (GLM), generalized additive model (GAM) and other statistical models (Pascal et al. 2014; Phung et al. 2018; Cai et al. 2019).

In the present study, we selected 15 chemical species and adopted GLM with a natural spline for analyzing the adverse effects of PM 2.5 constituent and source contributions on daily mortality. Death counts were modeled with either Poisson distribution or over-dispersed Poisson distribution according to their distribution in the study cities. We applied natural spline (ns) function of time and temperature for controlling both long-term and seasonal trends of death counts. Furthermore, we added $\mathrm{PM}_{2.5}$ as a covariate for adjustment of the effects by total $\mathrm{PM}_{2.5}$ mass. With respect to degrees of freedom (df) of the natural spline, we conducted sensitivity analyses by changing df from 2 to 10 per year to find the df minimizing the Akaike Information Criterion (Akaike 1974). Based on the sensitivity results we selected df=2 per year for temperature in all cities and df=2 per year for time was used in DJ, GJ and US while $\mathrm{df}=6$ per year was used for SL. Furthermore, we also examined the delayed effect, single-lag effect from on the day of exposure (lag 0 ) to seven days (lag 7) after exposure, because lagging effects of the exposure to $\mathrm{PM}_{2.5}$ have been steadily observed (Janssen et al. 2013; Chai et al. 2019; Li et al. 2021). The final model we constructed is following Eq (2).

$\ln \left(E\left[Y_{t}\right]\right)=\beta_{0}+\beta_{1} \square X_{t-I}+\beta_{2} \square P M M_{2.5}+\beta_{3} \square R H+\beta_{4} \square B P+\beta_{5} \square D O W$

$+n s($ time, $d f)+n s($ temperature, $d f)+\varepsilon(2)$ 
where, $Y t$ is the observed daily death counts on the day $t, X_{t-1}$ represents the daily mean concentration of $\mathrm{PM}_{2.5}$ chemical constituents at day $t$ to I ( $I=0,1$ $, 2 \ldots, 7) ; \mathrm{RH}, \mathrm{BP}, \mathrm{DOW}$ stands for daily average relative humidity and barometric pressure, day of week respectively; $\beta_{1}-\beta_{5}$ is the coefficient of $\mathrm{Xt}$, PM 2.5 , $\mathrm{RH}, \mathrm{BP}, \mathrm{DOW}$. After drawing $\beta_{1}$ from Eq (2), the relative risk (RR) was calculated by the following Eq (3).

$\mathrm{RR}=\mathrm{e}^{\beta 1}(3)$

All analyses were performed by using 'mgcv' package in statistical software R version 4.0.1 (http://www.R-project.org)

\section{Results And Discussion}

\section{Descriptive statistics of Death, Meteorological data and $\mathrm{PM}_{2.5}$ composition}

Tables 1 and 2 shows the descriptive statistics of explanatory variables for the four cities. Due to the largest population in SL, daily death counts were much larger in SL while those in the other cities were quite similar to each other. The proportions of CVD and RD death in total death in SL and DJ were comparable to those of the whole country (21.4\% and $10.8 \%)$ whereas the CVD and RD death in GJ (26.3\%, $15.8 \%)$ and US (30.8\% and $15.4 \%)$ accounted for higher proportions of total death.

Table 1

Summary of daily mortality data and meteorological conditions in SL, DJ, GJ and US during 2014-2018.

\begin{tabular}{|c|c|c|c|c|c|c|c|c|c|c|c|c|c|c|}
\hline SL & $\begin{array}{l}\text { Avg. } \\
\text { (S.D.) }\end{array}$ & Min. & $P_{25}$ & Median & $P_{75}$ & Max. & Unit & DJ & $\begin{array}{l}\text { Avg. } \\
\text { (S.D.) }\end{array}$ & Min. & $P_{25}$ & Median & $P_{75}$ & Max. \\
\hline NA & $\begin{array}{l}108 \\
(14)\end{array}$ & 69 & 98 & 107 & 115 & 182 & \multirow[t]{3}{*}{ counts } & NA & $17(5)$ & 5 & 14 & 17 & 20 & 33 \\
\hline CVD & $24(6)$ & 8 & 20 & 23 & 27 & 44 & & CVD & $4(2)$ & 0 & 2 & 3 & 5 & 11 \\
\hline $\mathrm{RD}$ & $11(4)$ & 2 & 8 & 10 & 13 & 29 & & $\mathrm{RD}$ & $2(2)$ & 0 & 1 & 2 & 3 & 8 \\
\hline Temp & $\begin{array}{l}13.3 \\
(10.8)\end{array}$ & -14.8 & 3.8 & 14.8 & 22.9 & 33.7 & ${ }^{\circ} \mathrm{C}$ & Temp & $\begin{array}{l}13.7 \\
(10.2)\end{array}$ & -12.7 & 4.7 & 14.9 & 22.5 & 33.4 \\
\hline $\mathrm{RH}$ & $\begin{array}{l}59.4 \\
(14.8)\end{array}$ & 21.8 & 48.4 & 59.5 & 69.5 & 99.8 & $\%$ & $\mathrm{RH}$ & $\begin{array}{l}69.8 \\
(13.8)\end{array}$ & 27.5 & 60.0 & 70.9 & 79.3 & 99.3 \\
\hline BP & $\begin{array}{l}1006.2 \\
(7.9)\end{array}$ & 981 & 999.8 & 1006.5 & 1012.6 & 1026.8 & $\mathrm{hPa}$ & $\mathrm{BP}$ & $\begin{array}{l}1008.6 \\
(7.9)\end{array}$ & 985.7 & 1002.3 & 1008.9 & 1015.0 & 1029.1 \\
\hline GJ & & & & & & & & US & & & & & & \\
\hline NA & $19(5)$ & 6 & 16 & 18 & 22 & 36 & \multirow[t]{3}{*}{ counts } & NA & $13(4)$ & 2 & 9.25 & 12 & 14 & 28 \\
\hline CVD & $5(3)$ & 0 & 3 & 4 & 6 & 13 & & CVD & $4(2)$ & 0 & 2 & 3 & 4 & 13 \\
\hline $\mathrm{RD}$ & $3(2)$ & 0 & 1 & 2 & 3 & 11 & & $\mathrm{RD}$ & $2(2)$ & 0 & 0 & 1 & 2 & 8 \\
\hline Temp & $\begin{array}{l}14.7 \\
(9.4)\end{array}$ & -9.5 & 6.3 & 15.75 & 22.7 & 32.0 & ${ }^{\circ} \mathrm{C}$ & Temp & $\begin{array}{l}14.7 \\
(8.7)\end{array}$ & -8.2 & 7.2 & 15.7 & 21.8 & 31.8 \\
\hline $\mathrm{RH}$ & $\begin{array}{l}69.0 \\
(15.2)\end{array}$ & 23.9 & 58.2 & 69.8 & 79.5 & 99.0 & $\%$ & $\mathrm{RH}$ & $\begin{array}{l}65.9 \\
(19.4)\end{array}$ & 16.8 & 50.9 & 68.7 & 81.3 & 99.8 \\
\hline $\mathrm{BP}$ & $\begin{array}{l}1008.2 \\
(7.8)\end{array}$ & 985.3 & 1001.9 & 1008.4 & 1014.4 & 1027.6 & $\mathrm{hPa}$ & $\mathrm{BP}$ & $\begin{array}{l}1008.7 \\
(8.1)\end{array}$ & 982.5 & 1002.6 & 1008.6 & 1014.6 & 1030 \\
\hline
\end{tabular}


Table 2

Summary of $\mathrm{PM}_{2.5}$ mass and chemical constituents concentration of study cities during 2014-2018.

\begin{tabular}{|c|c|c|c|c|c|c|c|c|c|c|c|c|c|c|}
\hline SL & Avg. (S.D.) & Min. & $P_{25}$ & Median & $P_{75}$ & Max. & unit & DJ & Avg. (S.D.) & Min. & $P_{25}$ & Median & $P_{75}$ & Max. \\
\hline $\mathrm{PM}_{2.5}$ & $27.7(17.2)$ & 1.0 & 16.5 & 23.6 & 33.8 & 153.4 & \multirow[t]{10}{*}{ 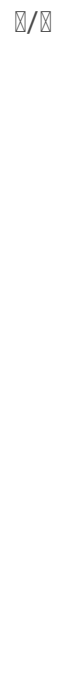 } & $\mathrm{PM}_{2.5}$ & $29.2(16)$ & 1.71 & 18.1 & 26.5 & 36.7 & 117.2 \\
\hline $\mathrm{SO}_{4}{ }^{2-}$ & $5.4(4.7)$ & 0.0 & 2.3 & 4.1 & 6.8 & 39.1 & & $\mathrm{SO}_{4}{ }^{2-}$ & $5.2(3.7)$ & 0.2 & 2.5 & 4.2 & 6.9 & 28.3 \\
\hline $\mathrm{NO}_{3}^{-}$ & $6.1(5.9)$ & 0.0 & 1.7 & 4.3 & 8.4 & 35.6 & & $\mathrm{NO}_{3}^{-}$ & $5.4(5.2)$ & 0.1 & 1.5 & 3.6 & 7.8 & 31.2 \\
\hline $\mathrm{Cl}^{-}$ & $0.3(0.4)$ & 0.0 & 0.1 & 0.2 & 0.5 & 2.6 & & $\mathrm{Cl}^{-}$ & $0.4(0.4)$ & 0.0 & 0.1 & 0.2 & 0.5 & 2.8 \\
\hline $\mathrm{Na}^{+}$ & $0.1(0.1)$ & 0.0 & - & 0.1 & 0.2 & 1.0 & & $\mathrm{Na}^{+}$ & $0.2(0.3)$ & 0.0 & 0.1 & 0.2 & 0.3 & 2.5 \\
\hline $\mathrm{NH}_{4}^{+}$ & $3.9(3.2)$ & 0.0 & 1.7 & 3.0 & 5.1 & 24.0 & & $\mathrm{NH}_{4}^{+}$ & $3.6(2.6)$ & 0.1 & 1.8 & 3.1 & 4.8 & 18.0 \\
\hline $\mathrm{K}^{+}$ & $0.2(0.2)$ & 0.0 & - & 0.1 & 0.2 & 1.0 & & $\mathrm{~K}^{+}$ & $0.2(0.2)$ & 0.0 & 0.1 & 0.1 & 0.2 & 1.5 \\
\hline $\mathrm{OC}$ & $3.8(1.9)$ & 0.3 & 2.5 & 3.4 & 4.7 & 16.8 & & $\mathrm{OC}$ & $4.5(2.1)$ & 0.6 & 3.0 & 4.2 & 5.7 & 16.5 \\
\hline EC & $1.3(0.6)$ & 0.1 & 0.8 & 1.1 & 1.6 & 4.5 & & $\mathrm{EC}$ & $1.4(0.7)$ & 0.3 & 0.8 & 1.2 & 1.7 & 4.9 \\
\hline S & 3.1 (2.3) & 0.1 & 1.5 & 2.4 & 3.9 & 23.3 & & $\mathrm{~S}$ & $3.9(3.9)$ & 0.1 & 1.5 & 2.6 & 4.8 & 40.1 \\
\hline $\mathrm{Ca}$ & $65.8(69.6)$ & 1.5 & 29.4 & 48.5 & 80.9 & 1105.0 & \multirow[t]{13}{*}{$\mathrm{ng} / \otimes$} & $\mathrm{Ca}$ & $58(73.8)$ & 1.5 & 16.5 & 36.4 & 75.5 & 1113 \\
\hline $\mathrm{Ti}$ & $9.8(7.8)$ & 0.5 & 5.4 & 8.3 & 12.1 & 106.5 & & $\mathrm{Ti}$ & $7.7(8)$ & 0.5 & 3.3 & 5.8 & 9.5 & 78.6 \\
\hline V & $3.7(4.6)$ & 0.3 & 0.3 & 1.8 & 5.0 & 31.6 & & V & $3.0(2.6)$ & 0.3 & 1.1 & 2.3 & 4.2 & 16.9 \\
\hline $\mathrm{Cr}$ & $1.3(1)$ & 0.3 & 0.3 & 1.1 & 1.7 & 7.5 & & $\mathrm{Cr}$ & $1.2(1.2)$ & 0.3 & 0.3 & 0.9 & 1.6 & 10.1 \\
\hline $\mathrm{Mn}$ & $12.3(8.5)$ & 0.3 & 6.5 & 10.1 & 15.7 & 56.1 & & $\mathrm{Mn}$ & $10(6.6)$ & 0.3 & 5.2 & 8.6 & 13.4 & 39.9 \\
\hline $\mathrm{Fe}$ & 195 (112.2) & 20.9 & 121 & 172.8 & 242.9 & 1074.5 & & $\mathrm{Fe}$ & 183 (113.9) & 16.6 & 103.7 & 160 & 234.3 & 1002.7 \\
\hline $\mathrm{Ni}$ & $1.6(1.6)$ & 0.2 & 0.4 & 1 & 2.2 & 10.1 & & $\mathrm{Ni}$ & $1.6(1.4)$ & 0.2 & 0.7 & 1.3 & 2.0 & 16.8 \\
\hline $\mathrm{Cu}$ & $7.5(5.2)$ & 1.0 & 3.8 & 6.6 & 9.8 & 35.0 & & $\mathrm{Cu}$ & $7(4.4)$ & 1.0 & 3.8 & 6.1 & 9.3 & 33.6 \\
\hline $\mathrm{Zn}$ & $65.5(43.6)$ & 2.5 & 35.4 & 54 & 82.7 & 302.3 & & $\mathrm{Zn}$ & $50.7(32)$ & 2.2 & 27.2 & 43.9 & 66 & 254.0 \\
\hline As & $4.0(3.3)$ & 0.3 & 1.7 & 3.4 & 5.6 & 25.7 & & As & $2.7(2.6)$ & 0.3 & 0.6 & 1.9 & 4.0 & 20.0 \\
\hline $\mathrm{Se}$ & $1.2(1.2)$ & 0.3 & 0.3 & 0.9 & 1.6 & 8.6 & & $\mathrm{Se}$ & $1.8(1.4)$ & 0.3 & 0.8 & 1.5 & 2.5 & 8.5 \\
\hline $\mathrm{Br}$ & $8.9(7)$ & 0.1 & 4.2 & 6.9 & 11.0 & 63.1 & & $\mathrm{Br}$ & $7.3(5.8)$ & 0.1 & 3.2 & 5.9 & 9.8 & 48.6 \\
\hline $\mathrm{Pb}$ & $21.8(15.7)$ & 0.7 & 11.9 & 18.2 & 27.7 & 132.9 & & $\mathrm{~Pb}$ & $18.8(13.9)$ & 0.7 & 8.7 & 15.3 & 24.7 & 115.3 \\
\hline
\end{tabular}


Table 2

(continued)

\begin{tabular}{|c|c|c|c|c|c|c|c|c|c|c|c|c|c|c|}
\hline GJ & Avg. (S.D.) & Min. & $P_{25}$ & Median & $P_{75}$ & Max. & unit & US & Avg. (S.D.) & Min. & $P_{25}$ & Median & $P_{75}$ & Max. \\
\hline $\mathrm{PM}_{2.5}$ & $26.9(15.8)$ & 3.3 & 16.2 & 23.5 & 33.5 & 132.9 & \multirow[t]{10}{*}{$\otimes / \otimes$} & $\mathrm{PM}_{2.5}$ & $20.4(11.8)$ & 2 & 11.7 & 17.8 & 26.1 & 88.3 \\
\hline $\mathrm{SO}_{4}^{2-}$ & $5.4(3.9)$ & 0.3 & 2.6 & 4.3 & 7.0 & 32.2 & & $\mathrm{SO}_{4}{ }^{2-}$ & $4.1(3.1)$ & 0.4 & 1.9 & 3.1 & 5.3 & 24.8 \\
\hline $\mathrm{NO}_{3}{ }^{-}$ & $4.6(4.8)$ & 0.1 & 1.4 & 3.0 & 6.3 & 41.1 & & $\mathrm{NO}_{3}^{-}$ & $3.0(3.0)$ & 0.1 & 0.9 & 2.0 & 3.9 & 21.8 \\
\hline $\mathrm{Cl}^{-}$ & $0.6(0.5)$ & 0.0 & 0.2 & 0.5 & 0.8 & 3.0 & & $\mathrm{Cl}^{-}$ & $0.2(0.2)$ & 0.0 & 0.1 & 0.2 & 0.3 & 1.4 \\
\hline $\mathrm{Na}^{+}$ & $0.2(0.1)$ & 0.0 & 0.1 & 0.1 & 0.2 & 1.3 & & $\mathrm{Na}^{+}$ & $0.1(0.1)$ & 0.0 & 0.1 & 0.1 & 0.2 & 0.8 \\
\hline $\mathrm{NH}_{4}^{+}$ & $3.7(2.5)$ & 0.2 & 1.9 & 3.1 & 4.7 & 19.3 & & $\mathrm{NH}_{4}^{+}$ & $2.5(1.8)$ & 0.2 & 1.2 & 2.1 & 3.3 & 14.5 \\
\hline $\mathrm{K}^{+}$ & $0.2(0.2)$ & 0.0 & 0.1 & 0.1 & 0.2 & 3.1 & & $\mathrm{~K}^{+}$ & $0.1(0.2)$ & 0.0 & 0.0 & 0.1 & 0.1 & 2.0 \\
\hline $\mathrm{OC}$ & $4.1(2.2)$ & 0.2 & 2.5 & 3.7 & 5.3 & 17.5 & & OC & $3.1(1.6)$ & 0.1 & 1.9 & 2.8 & 4.1 & 9.5 \\
\hline EC & $1.1(0.6)$ & 0.1 & 0.8 & 1.0 & 1.4 & 3.7 & & EC & $0.8(0.4)$ & 0.1 & 0.4 & 0.7 & 1.0 & 3.4 \\
\hline$S$ & $2.3(1.7)$ & 0.1 & 1.2 & 1.9 & 3.0 & 13.0 & & $S$ & $5.2(3.9)$ & 0.5 & 2.5 & 4.0 & 6.9 & 27.6 \\
\hline $\mathrm{Ca}$ & $58.1(81.8)$ & 1.5 & 18.9 & 33.0 & 63.2 & 912.6 & \multirow[t]{13}{*}{$\mathrm{ng} / \mathbb{\Delta}$} & $\mathrm{Ca}$ & $51.9(51)$ & 1.5 & 23.5 & 36.3 & 61.4 & 581.4 \\
\hline $\mathrm{Ti}$ & $8.5(9.7)$ & 0.5 & 3.6 & 5.6 & 9.7 & 103.9 & & $\mathrm{Ti}$ & $9.1(8.8)$ & 0.5 & 4.0 & 6.8 & 11.2 & 89.8 \\
\hline V & $3.5(3.1)$ & 0.3 & 1.3 & 2.8 & 4.8 & 23.5 & & V & $7.7(11)$ & 0.3 & 1.1 & 3.1 & 9.1 & 86.6 \\
\hline $\mathrm{Cr}$ & $0.8(0.7)$ & 0.3 & 0.3 & 0.3 & 1.1 & 7.1 & & $\mathrm{Cr}$ & $2.0(1.3)$ & 0.3 & 1.0 & 1.7 & 2.8 & 9.0 \\
\hline $\mathrm{Mn}$ & $11.9(7.9)$ & 0.3 & 6.4 & 10.3 & 15.5 & 52.6 & & $\mathrm{Mn}$ & $18.6(14.7)$ & 0.3 & 7.9 & 15.3 & 25.3 & 131.0 \\
\hline $\mathrm{Fe}$ & 177 (118.3) & 12.1 & 103.4 & 149.1 & 216.9 & 1129.8 & & $\mathrm{Fe}$ & 197 (128.4) & 10.3 & 109 & 169.8 & 255.9 & 1177.6 \\
\hline $\mathrm{Ni}$ & $1.3(1.1)$ & 0.2 & 0.5 & 1.0 & 1.7 & 9.8 & & $\mathrm{Ni}$ & $3.0(3.6)$ & 0.2 & 0.8 & 1.6 & 3.6 & 25.8 \\
\hline $\mathrm{Cu}$ & $4.0(3.6)$ & 1.0 & 2.1 & 3.3 & 5.1 & 46.3 & & $\mathrm{Cu}$ & $6.7(4.6)$ & 1.0 & 3.8 & 5.7 & 8.5 & 56.0 \\
\hline $\mathrm{Zn}$ & $57.1(35.3)$ & 2.4 & 32.2 & 48.7 & 73.2 & 230.4 & & $\mathrm{Zn}$ & $64.3(53.1)$ & 0.6 & 29.9 & 52 & 81.6 & 487.5 \\
\hline As & $3.7(2.2)$ & 0.3 & 2.1 & 3.3 & 4.7 & 16.0 & & As & $5.9(7.5)$ & 0.3 & 1.5 & 3.2 & 7.1 & 64.9 \\
\hline $\mathrm{Se}$ & $1.3(1.1)$ & 0.3 & 0.3 & 1.0 & 1.8 & 6.6 & & $\mathrm{Se}$ & $1.5(1.6)$ & 0.3 & 0.3 & 1.0 & 2.1 & 10.8 \\
\hline $\mathrm{Br}$ & $8.8(6.1)$ & 0.3 & 4.4 & 7.3 & 11.9 & 43.5 & & $\mathrm{Br}$ & $8.0(5.7)$ & 0.4 & 4.1 & 6.5 & 10.6 & 55.4 \\
\hline $\mathrm{Pb}$ & $19.7(17.3)$ & 0.7 & 7.5 & 15.0 & 26.9 & 115.4 & & $\mathrm{~Pb}$ & $17.7(15.4)$ & 0.7 & 7.1 & 13.4 & 23.6 & 121.7 \\
\hline
\end{tabular}

During the study period, the daily average temperature ( ${ }^{\circ} \mathrm{C}$ ) ranged from -14.8 to 33.7 (SL), -12.7 to 33.4 (DJ), -9.5 to 32.0 (GJ), -8.2 to 31.8 (US), which shows substantial temporal variation in all cities while the five-year average temperatures $\left({ }^{\circ} \mathrm{C}\right)$ were $13.3,13.7,14.7$ and 14.7 in $\mathrm{SL}, \mathrm{DJ}, \mathrm{GJ}$ and $\mathrm{US}$ respectively. The daily average relative humidity (\%) ranged 21.8-99.8, 27.5-99.3, 23.9-99.0 and 16.8-99.8 in SL, DJ, GJ and US respectively.

The average concentrations of $\mathrm{PM}_{2.5}$ mass at all sites exceeded both World Health Organization guidelines $\left(5 \mu \mathrm{g} / \mathrm{m}^{3}\right.$ of annual mean concentration) and U.S. Environment Protection Agency standards $\left(15 \mu \mathrm{g} / \mathrm{m}^{3}\right.$ of annual mean concentration). This suggests the population in four cities have been exposed to potential health risk during 2014-2018. It is notable that Ulsan showed approximately $25-30 \%$ lower $\mathrm{PM}_{2.5}$ concentration than the other cities.

$\mathrm{PM}_{2.5}$ chemical compositions showed considerable spatial variability. Secondary inorganic aerosols $\left(\mathrm{SIA}^{2}\right.$ i.e., $\left.\mathrm{SO}_{4}{ }^{2-}, \mathrm{NO}_{3}{ }^{-}, \mathrm{NH}_{4}{ }^{+}\right)$constructed the total mass at a range of $46.9 \%$ (US)-55.6\% (SL), which indicates secondary aerosol formation were a important factor in $\mathrm{PM}_{2.5}$. In addition, the $\mathrm{SO}_{4}{ }^{2-} / \mathrm{NO}_{3}{ }^{-}$ ratio was much lower in SL (0.88) than those in DJ (0.97), GJ (1.16) and US (1.38), which implies the dominant gas-phase pollutants were different across the cities. Carbonaceous species, reported to have second largest proportions in $\mathrm{PM}_{2.5}$ mass in South Korea (Son et al. 2012; Bae et al. 2020), accounted for $18.2 \%(\mathrm{SL}), 20.1 \%$ (DJ), $19.6 \%$ (GJ) and $18.4 \%$ (US) respectively. The proportions of trace elements were much higher in US (27.4\%) than in SL (12.5\%), DJ (14.5\%) and GJ (10.0\%), considered to reflect the industrial characteristics of US.

\section{Source apportionment $\mathrm{PM}_{2.5}$}


A total of $1,438,1,278,1,419$ and 1,211 $\mathrm{PM}_{2.5}$ speciation datasets were used for identifying $\mathrm{PM}_{2.5}$ sources and their contributions for SL, DJ, GJ and US respectively. We chose the number of factors based on the evaluation of model results including Q-value, residual distribution and the coefficient of determination and we confirmed source profiles in the study cities were physically meaningful and understandable.

Nine-factor solution for DJ and ten-factor solution for SL, GJ and US solution were drawn while source contributions were quite different among cities although the resolved factors were identical. Sources and their contributions to $\mathrm{PM}_{2.5}$ are listed in Table 3 and source profiles for SL, DJ, GJ and US obtained from PMF model are displayed in Figures S1-S4 respectively.

Table 3

Sources with their contributions to $\mathrm{PM}_{2.5}$ in SL, DJ, GJ and US during 2014-2018

\begin{tabular}{|lllllllll|}
\hline Source & $\mathrm{SL}$ & \multicolumn{3}{c}{ DJ } & \multicolumn{3}{c|}{ GJ } & \multicolumn{3}{c|}{ US } \\
\cline { 2 - 10 } & $\boldsymbol{\mu g} / \mathrm{m}^{3}$ & $\%$ & $\mu \mathrm{g} / \mathrm{m}^{3}$ & $\%$ & $\mu \mathrm{g} / \mathrm{m}^{3}$ & $\%$ & $\mu \mathrm{g} / \mathrm{m}^{3}$ & $\%$ \\
\hline Secondary nitrate & 6.4 & 20.3 & 5.7 & 19.5 & 5.2 & 19.5 & 3.6 & 18.9 \\
\hline Secondary sulfate & 6.9 & 21.8 & 5.7 & 19.7 & 5.7 & 21.3 & 4.5 & 23.9 \\
\hline Mobile & 7.1 & 22.3 & 6.3 & 21.7 & 5.5 & 20.5 & 4.7 & 24.9 \\
\hline Biomass burning & 1.4 & 4.3 & 0.8 & 2.7 & 0.9 & 3.2 & 0.7 & 3.6 \\
\hline District heating & 2.8 & 8.7 & 2.6 & 8.9 & 3.3 & 12.2 & 0.7 & 3.7 \\
\hline Soil & 0.8 & 2.6 & 1.2 & 4.2 & 1.1 & 4.2 & 1.0 & 5.3 \\
\hline Industry & 1.4 & 4.4 & 1.9 & 6.6 & 1.2 & 4.3 & 1.7 & 8.8 \\
\hline Coal combustion & 3.4 & 10.7 & 3.1 & 10.7 & 2.6 & 9.7 & 0.7 & 3.5 \\
\hline Oil combustion & 0.9 & 2.9 & 1.8 & 6.1 & 0.6 & 2.2 & 0.6 & 3.4 \\
\hline Aged sea salt & 0.7 & 2.1 & - & - & 0.8 & 2.9 & 0.7 & 3.9 \\
\hline
\end{tabular}

In SL, secondary nitrate, secondary sulfate and mobile source made up the majority (64.4\%) of the PM 2.5 mass concentration, consistent with the past studies conducted for SL (Heo et al. 2009; Moon et al., 2010). Mobile was the largest contributor followed by secondary sulfate, secondary nitrate and coal combustion, which implies sources related to secondary formation were dominant for $\mathrm{PM}_{2.5}$. Especially sulfur related sources, secondary sulfate and coal combustion, were thought to be affected by several huge coal-fired power plants (e.g., Taean 6100 MW, Dangjin 6040 MW, Yeongheung 5080 MW) located in the southwest part of the city. These results are supported by previous studies which also identified those coal power stations as attributable factors to $\mathrm{PM}_{2.5}$ in SL by analyzing polycyclic aromatic hydrocarbons (Kang et. al. 2020) and executing dispersion modelling (Kim et. al. 2016).

Similar to SL, $\mathrm{PM}_{2.5}$ mass concentration in DJ was mostly contributed by four factors; mobile (21.7\%), secondary sulfate (19.7\%), secondary nitrate (19.5\%) and coal combustion (10.7\%) while aged sea salt source was not solely resolved due to the location in deep land. In GJ secondary sulfate (21.3\%) was the largest contributor followed by mobile (20.5\%) and secondary nitrate (19.5\%) source and those major factors in total explained $61.3 \%$ of $\mathrm{PM}_{2.5}$ mass concentration.

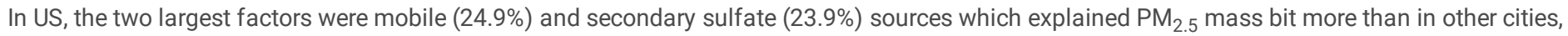
assumed to reflect the characteristics of the city where both huge heavy industrial complex and industrial ports exist. The annual emission of SOx in the city during 2014-2017 was 48,433 ton on average more than two times of the national average annual emission (19,516 ton). In the same context the contribution of industry (8.8\%) was much bigger than that in the other cities and it was fourth-largest factor in the city.

\section{Association of PM 2.5 chemical constituents and source contributions with mortality}

Using PMF-modeled source contributions as well as $\mathrm{PM}_{2.5}$ speciation data, we analyzed the effects of the $\mathrm{PM}_{2.5}$ chemical constituents of $\mathrm{PM} \mathrm{C}_{2.5}$ and sources on cause-specific mortality. Overall, the IQR increase in the concentration of each constituents influenced all cause-specific mortality whereas only a few constituents were significantly associated with the mortality with different strength depending on city and cause of death, which shows risk heterogeneity among various $\mathrm{PM}_{2.5}$ constituents. In addition, the impacts of source contributions on mortality also varied among the cities. The associations of cause-specific mortality with both $\mathrm{PM}_{2.5}$ constituents and sources are summarized in Tables S1-S2 respectively and Table 4 shows the highest RR of mortality significantly associated with chemical constituents. 
Table 4

The highest $\mathrm{RR}$ and $95 \% \mathrm{Cl}$ of the significant associations of cause-specific mortality with $\mathrm{PM}_{2.5}$ constituents in SL, DJ, GJ and US.

\begin{tabular}{|c|c|c|c|c|c|c|c|c|c|}
\hline City & Cause & Constituents & Lags & Constituents & Lags & Constituents & Lags & Constituents & Lags \\
\hline \multirow[t]{6}{*}{ SL } & NA & $\mathrm{K}^{+}(1.016)$ & 7 & & 7 & & 2 & - & - \\
\hline & & $(1.002,1.031)$ & & $(1.003,1.019)$ & & $(1.000,1.031)$ & & & \\
\hline & CVD & $\mathrm{Ti}(1.016)$ & 7 & $\mathrm{Fe}(1.023)$ & 7 & As (1.030) & 1 & - & - \\
\hline & & $(1.001,1.032)$ & & $(1.003,1.044)$ & & $(1.003,1.058)$ & & & \\
\hline & $\mathrm{RD}$ & $\mathrm{Pb}(1.071)$ & 0 & - & - & - & - & - & - \\
\hline & & $(1.013,1.128)$ & & & & & & & \\
\hline \multirow[t]{8}{*}{ DJ } & NA & EC (1.051) & 2 & $\mathrm{Fe}(1.039)$ & 2 & - & - & - & - \\
\hline & & $(1.004,1.100)$ & & $(1.009,1.069)$ & & & & & \\
\hline & CVD & OC (1.146) & 4 & V (1.080) & 1 & Mn (1.097) & 1 & $\mathrm{Ni}(1.042)$ & 1 \\
\hline & & $(1.057,1.239)$ & & $(1.007,1.154)$ & & $(1.027,1.167)$ & & $(1.005,1.080)$ & \\
\hline & & Zn (1.076) & 1 & $\mathrm{~Pb}(1.091)$ & 1 & - & & - & - \\
\hline & & $(1.005,1.148)$ & & $(1.006,1.177)$ & & & & & \\
\hline & $\mathrm{RD}$ & OC (1.219) & 5 & As (1.190) & 0 & - & & - & - \\
\hline & & $(1.090,1.354)$ & & $(1.007,1.384)$ & & & & & \\
\hline \multirow[t]{6}{*}{ GJ } & NA & Ti (1.015) & 3 & - & - & - & - & - & - \\
\hline & & $(1.001,1.029)$ & & & & & & & \\
\hline & $\mathrm{RD}$ & $\mathrm{SO}_{4}^{2-}(1.085)$ & 1 & $\mathrm{NH}_{4}^{+}(1.071)$ & 1 & Ti (1.048) & 5 & V (1.109) & 1 \\
\hline & & $(1.009,1.163)$ & & & & $(1.009,1.088)$ & & $(1.040,1.179)$ & \\
\hline & & $\mathrm{Fe}(1.061)$ & 5 & $\mathrm{Ni}(1.100)$ & 1 & Zn (1.078) & 1 & - & - \\
\hline & & $(1.001,1.120)$ & & (1.033 1.170) & & $(1.010,1.146)$ & & & \\
\hline \multirow[t]{4}{*}{ US } & NA & $\mathrm{SO}_{4}^{2-}(1.047)$ & 4 & OC (1.056) & 4 & $\operatorname{Mn}(1.060)$ & 0 & - & - \\
\hline & & $(1.004,1.091)$ & & $(1.001,1.113)$ & & $(1.018,1.103)$ & & & \\
\hline & CVD & OC (1.126) & 4 & - & - & - & - & - & - \\
\hline & & $(1.009,1.248)$ & & & & & & & \\
\hline
\end{tabular}

In SL, the chemical constituents showing significant associations were as follows: $\mathrm{K}^{+}(\mathrm{RR} 1.016,95 \% \mathrm{Cl} 1.002-1.032), \mathrm{EC}(1.015,1.000-1.031)$ and Ti (1.011, 1.003-1.019) with NA mortality, Ti (1.016, 1.001-1.032), Fe (1.023, 1.003-1.044) and As (1.030, 1.003-1.058) with CVD mortality and Pb (1.071, 1.013-1.128) with RD mortality. Ti and Fe, mostly originated from soil dust, are known to generate reactive oxygen species which lead to cell damage (Moreno et al. 2019). As and Pb, markers of coal combustion, are toxic even at low levels and they are known as carcinogen to human by International Agency for Research on Cancer. Significant associations of mortality with sources were mostly found for CVD mortality: district heating (1.032, 1.0071.057), coal combustion (1.034, 1.003-1.065), soil (1.016, 1.000-1.032). Overall, the results in SL showed that the health effects were closely linked to transition metals from both natural and combustion-derived sources. Transition metals have impacts on cardiovascular via both direct and indirect pathways inducing oxidative stress and inflammation (Mills et al. 2009). In addition, respiratory system is subject to damage by those metals which trigger oxidative stress in lung alveoli (Donaldson et al. 1996).

In DJ we found $\mathrm{PM}_{2.5}$ constituents increased the RR of all types of mortality but most of the significant associations were related to CVD mortality. Constituents showing significant associations were EC (1.051, 1.004-1.100) and Fe (1.039, 1.009-1.069) with NA mortality, OC (1.146, 1.057-1.239), V (1.080, 1.007-1.154), Mn (1.097, 1.027-1.167), Ni (1.042, 1.005-1.080), Zn (1.076, 1.005-1.148) and Pb (1.091, 1.006-1.177) with CVD mortality and OC $(1.219,1.090-1.354)$ and As $(1.190,1.007-1.384)$ with RD mortality. Beside transition metals mentioned earlier, OC and EC have been consistently reported to influence on various indicators of cardiovascular disease such as blood pressure, heart rate variability (Huang et al. 2012; Schneider et al. 2012; Wu et al. 2013) which can lead to cardiovascular health outcomes (Bell et al. 2009; Ito et al. 2011). Accordingly, sources with carbonaceous species and transition metals were thought to be major factors associated with mortality in DJ. 
Significant associations in GJ were mostly found for RD mortality, which was uniquely observed among the four cities. Chemical species significantly associated with $\mathrm{RD}$ mortality were ionic species, $\mathrm{SO}_{4}{ }^{2-}(1.085,1.009-1.163), \mathrm{NH}_{4}{ }^{+}(1.071,1.000-1.143)$, as well as transition metals including $\mathrm{Ti}(1.048$, 1.009-1.088), V (1.109, 1.040-1.179), Fe (1.061, 1.001-1.120), Ni (1.100, 1.033-1.170), Zn (1.078, 1.010-1.146). While several previous studies revealed the significant association $\mathrm{SO}_{4}{ }^{2-}$ with the risk of mortality, there are not explicit biological mechanisms of $\mathrm{SO}_{4}{ }^{2-}\left(\mathrm{Ueda}^{-}\right.$et al. 2016$)$. Nevertheless, some plausible theories have been suggested to explain the positive association of $\mathrm{SO}_{4}{ }^{2-}$ with adverse health effects. First, particle acidity by $\mathrm{SO}_{4}{ }^{2-}$ may change the pulmonary toxicity of other $\mathrm{PM}_{2.5}$ constituents or physical properties from their own toxicity (Dreher 2000). Second, catalyzation of metals into more bioavailable forms is another possible explanation for the high associations of $\mathrm{SO}_{4}{ }^{2-}$ with health effects (Lay et al. 2001). Regarding source contributions, sources of the species with significant association (e.g., coal combustion, mobile) were revealed to increase the RD mortality in GJ.

In US, $\mathrm{SO}_{4}{ }^{2-}(1.047,1.004-1.091), \mathrm{OC}(1.056,1.001-1.113)$ and Mn $(1.060,1.018-1.103)$ for NA mortality and OC (1.126, 1.009-1.248) for CVD mortality increased the RR significantly. In addition, secondary sulfate (1.052, 1.007-1.098), mobile (1.053, 1.010-1.098) and industry (1.059, 1.017-1.102) sources were strongly associated with NA or RD mortality. These results were in consistent with the source profiles from PMF modelling which identified higher contribution of mobile and secondary sulfate and the composition of $\mathrm{PM}_{2.5}$ compositional characteristic with the highest $\mathrm{SO}_{4}{ }^{2-} / \mathrm{NO}_{3}{ }^{-}$ratio among four cities.

\section{Conclusion}

In the present study, we investigated the associations of cause-specific mortality with the IQR increase in the concentration of PM 2.5 constituents and source contributions in four metropolitan cities in South Korea. The significance and strength of associations varied across the study cities, which implied the adverse health effects of short-term exposure to $\mathrm{PM}_{2.5}$ were spatially heterogeneous. In SL significant associations were found for both NA and CVD mortality mostly related to transition metals and relevant sources including soil and coal combustion. In DJ most of the significant associations were found for CVD mortality with $\mathrm{PM}_{2.5}$ constituents from combustion related sources including mobile, oil combustion and industry. However, many of significant associations in $\mathrm{GJ}$ were found for $\mathrm{RD}$ mortality with constituents related to secondary formation (e.g., $\mathrm{SO}_{4}{ }^{2-}$, $\left.\mathrm{NH}_{4}{ }^{+}\right)$and heavy metals. Lastly, we found significant associations in US were closely related to $\mathrm{SO}_{4}{ }^{2-}, \mathrm{OC}$ and $\mathrm{Mn}$ in line with the characteristic of the city where large heavy and chemical industry complexes are located in.

From the results, we drew a conclusion that the risk of mortality increased by short-term exposure to PM 2.5 were quite heterogeneous. Therefore, identifying $\mathrm{PM}_{2.5}$ constituents and source contributions affecting health outcomes significantly in the region of interest should takes priority over designing policies for controlling $\mathrm{PM}_{2.5}$ efficiently. In addition, the policies, currently focused on $\mathrm{PM}_{2.5}$ mass concentration, need to be shifted to health risk-based ones for protecting public health from air pollution.

There is still limitation of the present study to be improved in further studies. Although ambient $\mathrm{PM}_{2.5}$ compositional data was employed as a proxy of personal exposure to $\mathrm{PM}_{2.5}$ and we acquired the data from one site for each city due to the insufficient number of APIMS. As the area one APIMS cover is broader, the exactness of estimating the level of $\mathrm{PM}_{2.5}$ exposure decrease, which may result in inevitable errors in evaluating the health effects. Accordingly, more sites for $\mathrm{PM}_{2.5}$ speciation need to be established considering relevant indexes such as population, administrative area for a better assessment of $\mathrm{PM}_{2.5}$ exposure.

\section{Declarations}

Ethics approval and consent to participate Not applicable.

Consent for publication Not applicable.

Data Availability All data generated or analyzed during this study are included in this published article and its supplementary information files.

Competing interests The authors declare that they have no competing interests

Funding Not applicable.

Authors' contributions Ho Kim and Seung-Muk Yi supervised study design; Inho Song, Dae-Gon Kim and Kwonho Jeon contributed to data collection and verification; Sangcheol Kim, Juyeon Yang performed data analyses and wrote the first draft of the manuscript; Sangcheol Kim, Juyeon Yang, Jieun Park, Inho Song, Dae-Gon Kim, Kwonho Jeon, Ho Kim and Seung-Muk Yi contributed to interpretation of the data. All authors read and approved the final manuscript.

Acknowledgements This work was supported by the National Institute of Environmental Research (NIER) of the Ministry of Environment under grant No. NIER-2021-03-03-001 and NIER-2020-04-02-086 and the National Strategic Project-Fine particle (NRF-2017M3D8A1092019) of the National Research Foundation of Korea (NRF)

\section{References}


1. Akaike H (1974) A new look at the statistical model identification. IEEE transactions on automatic control 19:716-723. 10.1109/TAC.1974.1100705

2. Allen MJ, Sheridan SC (2014) High-mortality days during the winter season: comparing meteorological conditions across 5 US cities. Int $J$ Biometeorol 58:217-225. https://doi.org/10.1007/s00484-013-0640-4

3. Amil N, Latif MT, Khan MF, Mohamad M (2016) Seasonal variability of $\mathrm{PM}_{2.5}$ composition and sources in the Klang Valley urban-industrial environment. Atmos Chem Phys 16(8):5357-5381. https://doi.org/10.5194/acp-16-5357-2016

4. Atkinson RW, Kang S, Anderson HR, Mills IC, Walton HA (2014) Epidemiological time series studies of $\mathrm{PM}_{2.5}$ and daily mortality and hospital admissions: a systematic review and meta-analysis. Thorax 69:660-665. http://dx.doi.org/10.1136/thoraxjnl-2013-204492

5. Bae C, Kim BU, Kim HC, Yoo C, Kim S (2020) Long-range transport influence on key chemical components of PM 2.5 in the Seoul Metropolitan Area, South Korea, during the years 2012-2016. Atmosphere 11:48. https://doi.org/10.3390/atmos11010048

6. Begum BA, Biswas SK, Hopke PK (2007) Source Apportionment of Air Particulate Matter by Chemical Mass Balance (CMB) and Comparison with Positive Matrix Factorization (PMF) Model. Aerosol Air Qual Res 7:446-468. https://doi.org/10.4209/aaqr.2006.10.0021

7. Bell ML, Ebisu K, Peng RD, Samet JM, Dominici F (2009) Hospital admissions and chemical composition of fine particle air pollution. Am J Respir Crit Care Med 179:1115-1120. https://doi.org/10.1164/rccm.200808-12400C

8. Cai J, Peng C, Yu S, Pei Y, Liu N, Wu Y, Fu Y, Cheng J (2019) Association between PM $_{2.5}$ exposure and all-cause, non-accidental, accidental, different RD diseases, sex and age mortality in Shenzhen, China. Int J Environ Res Public Health 16:401. https://doi.org/10.3390/ijerph16030401

9. Chai G, He H, Sha Y, Zhai G, Zong S (2019) Effect of $\mathrm{PM}_{2.5}$ on daily outpatient visits for respiratory diseases in Lanzhou, China. Sci Total Environ 649:1563-1572. https://doi.org/10.1016/j.scitotenv.2018.08.384

10. Cheng MC, You CF, Cao J, Jin Z (2012) Spatial and seasonal variability of water-soluble ions in $\mathrm{PM}_{2.5}$ aerosols in 14 major cities in China. Atmos Environ 60:182-192. https://doi.org/10.1016/j.atmosenv.2012.06.037

11. Churg A, Brauer M (1997) Human lung parenchyma retains $\mathrm{PM}_{2.5}$. Am J Respir Crit Care Med 155(6):2109-2111. https://doi.org/10.1164/ajrccm.155.6.9196123

12. Dai W, Gao J, Cao G, Ouyang F (2013) Chemical composition and source identification of $\mathrm{PM}_{2.5}$ in the suburb of Shenzhen, China. Atmos Res 122:391-400. https://doi.org/10.1016/j.atmosres.2012.12.004

13. Donaldson K, Beswick PH, Gilmour PS (1996) Free radical activity associated with the surface of particles: a unifying factor in determining biological activity. Toxicol Lett 88:293-298. https://doi.org/10.1016/0378-4274(96)03752-6

14. Dreher KL (2000) Particulate matter physicochemistry and toxicology: In search of causality-A critical perspective. Inhal Toxicol $12: 45-57$. https://doi.org/10.1080/08958378.2000.11463230

15. Ghio AJ, Stoneheurner J, McGee JK, Kinsey JS (1999) SULFATE CONTENT CORRELATES WITH IRON CONCENTRATIONS IN AMBIENT AIR POLLUTION PARTICLES. Inhal Toxicol 11:293-307. DOI:10.1080/089583799197104

16. He K, Zhao Q, Ma Y, Duan F, Yang F, Shi Z, Chen G (2012) Spatial and seasonal variability of PM 2.5 acidity at two Chinese megacities: insights into the formation of secondary inorganic aerosols. Atmos Chem Phys 12:1377-1395. https://doi.org/10.5194/acp-12-1377-2012

17. Heo JB, Hopke PK, Yi SM (2009) Source apportionment of $\mathrm{PM}_{2.5}$ in Seoul, Korea. Atmos Chem Phys 9:4957-4971. https://doi.org/10.5194/acp-94957-2009

18. Huang W, Zhu T, Pan X, Hu M, Lu SE, Lin Y, Wang T, Zhang Y, Tang X (2012) Air pollution and autonomic and vascular dysfunction in patients with cardiovascular disease: interactions of systemic inflammation, overweight, and gender. Am J Epidemiol 176:117-126. https://doi.org/10.1093/aje/kwr511

19. Ito K, Mathes R, Ross Z, Nadas A, Thurston G, Matte T (2011) Fine particulate matter constituents associated with cardiovascular hospitalizations and mortality in New York City. Environ Health Perspect 119:467-473. https://doi.org/10.1289/ehp.1002667

20. Janssen NAH, Fischer P, Marra M, Ameling C, Cassee FR (2013) Short-term effects of $\mathrm{PM}_{2.5}, \mathrm{PM}_{10}$ and $\mathrm{PM}_{2.5-10}$ on daily mortality in the Netherlands. Sci Total Environ 463:20-26. https://doi.org/10.1016/j.scitotenv.2013.05.062

21. Kang M, Kim K, Choi N, Kim YP, Lee JY (2020) Recent Occurrence of PAHs and n-Alkanes in $\mathrm{PM}_{2.5}$ in Seoul, Korea and Characteristics of Their Sources and toxicity. Int J Environ Res Public Health 17:1397. https://doi.org/10.3390/ijerph17041397

22. Ke L, Liu W, Wang Y, Russell AG, Edgerton ES, Zheng M (2008) Comparison of $\mathrm{PM}_{2.5}$ source apportionment using positive matrix factorization and molecular marker-based chemical mass balance. Sci Total Environ 394:290-302. https://doi.org/10.1016/j.scitotenv.2008.01.030

23. Kim BU, Kim O, Kim HC, Kim S (2016) Influence of fossil-fuel power plant emissions on the surface fine particulate matter in the Seoul Capital Area, South Korea. J Air Waste Manag Assoc 66:863-873. https://doi.org/10.1080/10962247.2016.1175392

24. Kim HW, Dong L, Choi AES, Fujii M, Fujita T, Park HS (2018) Co-benefit potential of industrial and urban symbiosis using waste heat from industrial park in Ulsan, Korea. Resour Conserv Recycl 135:225-234. https://doi.org/10.1016/j.resconrec.2017.09.027

25. Kong S, Ding X, Bai Z, Han B, Chen L, Shi J, Li Z (2010) A seasonal study of polycyclic aromatic hydrocarbons in $\mathrm{PM}_{2.5}$ and PM $2.5-10$ in five typical cities of Liaoning Province, China. J Hazard Mater 183:70-80. https://doi.org/10.1016/j.jhazmat.2010.06.107 
26. Lay JC, Zeman KL, Ghio AJ, Bennett WD (2001) Effects of inhaled iron oxide particles on alveolar epithelial permeability in normal subjects. Inhal Toxicol 13:1065-1078

27. Li X, Chen X, Yuan X, Zeng G, León T, Liang J, Chen G, Yuan X (2017) Characteristics of particulate pollution ( $P M_{2.5}$ and $\left.P M_{10}\right)$ and their spacescaledependent relationships with meteorological elements in China. Sustainability 9:2330. https://doi.org/10.3390/su9122330

28. Li B, Yang J, Dong H, Li M, Cai D, Yang Z, Zhang C, Wang H, Hu J, Bergmann S, Lin G, Wang B (2021) PM 2.5 constituents and mortality from a spectrum of causes in Guangzhou, China. Ecotoxicol Environ Saf 222:112498. https://doi.org/10.1016/j.ecoenv.2021.112498

29. Manousakas M, Papaefthymiou H, Diapouli E, Migliori A, Karydas AG, Bogdanovic-Radovic I, Eleftheriadis K (2017) Assessment of PM 2.5 sources and their corresponding level of uncertainty in a coastal urban area using EPA PMF 5.0 enhanced diagnostics. Sci Total Environ 574:155-164. https://doi.org/10.1016/j.scitotenv.2016.09.047

30. Mills NL, Donaldson K, Hadoke PW, Boon NA, MacNee W, Cassee FR, Sandström T, Blomberg A, Newby DE (2009) Adverse cardiovascular effects of air pollution. Nat Clin Pract Cardiovasc Med 6:36-44. https://doi.org/10.1038/ncpcardio1399

31. Moon KJ, Park SM, Park JS, Song IH, Jang SK, Kim JC, Lee SJ (2011) Chemical Characteristics and Source Apportionment of PM 2.5 in Seoul Metropolitan Area in 2010. J Korean Soc Atmos Environ 27:711-722. https://doi.org/10.5572/KOSAE.2011.27.6.711.(in Korean)

32. Moreno T, Trechera P, Querol X, Lah R, Johnson D, Wrana A, Williamson B (2019) Trace element fractionation between $P_{10}$ and $P M_{2.5}$ in coal mine dust: Implications for occupational RD health. Int J Coal Geol 203:52-59. https://doi.org/10.1016/j.coal.2019.01.006

33. Norris G, Duvall R, Brown S, Bai S (2008) EPA Positive Matrix Factorization (PMF) 5.0 Fundamentals and User Guide. Prepared for the US Environmental Protection Agency Office of Research and Development, Washington, DC. DC EPA/600/R-14/108

34. Paatero P, Eberly S, Brown SG, Norris GA (2014) Methods for estimating uncertainty in factor analytic solutions. Atmos Meas Tech 7:781-797. https://doi.org/10.5194/amt-7-781-2014

35. Pandey P, Patel DK, Khan AH, Barman SC, Murthy RC, Kisku GC (2013) Temporal distribution of fine particulates $\left(\mathrm{PM}_{2.5}, \mathrm{PM}_{10}\right)$, potentially toxic metals, PAHs and Metal-bound carcinogenic risk in the population of Lucknow City, India. J Environ Sci Health 48:730-745. https://doi.org/10.1080/10934529.2013.744613

36. Pascal M, Falq G, Wagner V, Chatignoux E, Corso M, Blanchard M, Host S, Pascal L, Larrieu S (2014) Short-term impacts of particulate matter (PM 10 , $\mathrm{PM}_{10-2.5}, \mathrm{PM}_{2.5}$ ) on mortality in nine French cities. Atmos Environ 95:175-184. https://doi.org/10.1016/j.atmosenv.2014.06.030

37. Phung VLH, Ueda K, Kasaoka S, Seposo X, Tasmin S, Yonemochi S, Phosri A, Honda A, Takano H, Michikawa T, Nitta H (2018) Acute effects of ambient $\mathrm{PM}_{2.5}$ on all-cause and cause-specific emergency ambulance dispatches in Japan. Int J Environ Res Public Health 15:307. https://doi.org/10.3390/ijerph15020307

38. Pradeau C, Rondeau V, Leveque E, Guernion PY, Tentillier E, Thicoipe M, Brochard P (2015) Air pollution and activation of mobile medical team for out-of-hospital cardiac arrest. Am J Emerg Med 33:367-372. https://doi.org/10.1016/j.ajem.2014.12.007

39. Qiao L, Cai J, Wang H, Wang W, Zhou M, Lou S, Chen R, Dai H, Chen C, Kan H (2014) $\mathrm{PM}_{2.5}$ constituents and hospital emergency-room visits in Shanghai, China. Environ Sci Technol 48:10406-10414. https://doi.org/10.1021/es501305k

40. Schins RPF, Lightbody JH, Borm PJA, Shi T, Donaldson K, Stone V (2004) Inflammatory effects of coarse and fine particulate matter in relation to chemical and biological constituents. Toxicol Appl Pharmacol 195:1-11. https://doi.org/10.1016/j.taap.2003.10.002

41. Schneider A, Hampel R, Ibald-Mulli A, Zareba W, Schmidt G, Schneider R, Rückerl R, Couderc JP, Mykins B, Oberdörster G, Wölke G, Pitz M, Wichmann $\mathrm{H}-\mathrm{E}$, Peters $\mathrm{A}(2010)$ Changes in deceleration capacity of heart rate and heart rate variability induced by ambient air pollution in individuals with coronary artery disease. Part Fibre Toxicol 7:1-12. https://doi.org/10.1186/1743-8977-7-29

42. Son JY, Lee JT, Kim KH, Jung K, Bell ML (2012) Characterization of fine particulate matter and associations between particulate chemical constituents and mortality in Seoul, Korea. Environ Health Perspect 120:872-878. https://doi.org/10.1289/ehp.1104316

43. Song Y, Zhang Y, Xie S, Zeng L, Zheng M, Salmon LG, Shao M, Slanina S (2006) Source apportionment of PM 2.5 in Beijing by positive matrix factorization. Atmos Environ 40:1526-1537. https://doi.org/10.1016/j.atmosenv.2005.10.039

44. Teixeira EC, Agudelo-Castaneda DM, Mattiuzi CDP (2015) Contribution of polycyclic aromatic hydrocarbon (PAH) sources to the urban environment: A comparison of receptor models. Sci Total Environ 538:212-219. https://doi.org/10.1016/j.scitotenv.2015.07.072

45. Ueda K, Yamagami M, Ikemori F, Hisatsune K, Nitta H (2016) Associations between fine particulate matter components and daily mortality in Nagoya, Japan. Epidemiol J, JE20150039. https://doi.org/10.2188/jea.JE20150039

46. Wang-Li L (2015) Insights to the formation of secondary inorganic $\mathrm{PM}_{2.5}$ : Current knowledge and future needs. International Journal of Agricultural Biological Engineering 8:1-13. DOI:10.3965/j.jjabe.20150802.1810

47. Wang Y, Shi Z, Shen F, Sun J, Huang L, Zhang H, Chen C, Li T, Hu J (2019) Associations of daily mortality with short-term exposure to PM 2.5 and its constituents in Shanghai, China. Chemosphere 233:879-887. https://doi.org/10.1016/j.chemosphere.2019.05.249

48. Watson JG, Antony Chen LW, Chow JC, Doraiswamy P, Lowenthal DH (2008) Source apportionment: findings from the US supersites program. J Air Waste Manag Assoc 58:265-288. https://doi.org/10.3155/1047-3289.58.2.265

49. Wilson WE, Suh HH (1997) Fine particles and coarse particles: concentration relationships relevant to epidemiologic studies. J Air Waste Manag Assoc 47:1238-1249. https://doi.org/10.1080/10473289.1997.10464074

Page $11 / 12$ 
50. Wu S, Deng F, Huang J, Wang H, Shima M, Wang X, Qin Y, Zheng C, Wei H, Hao Y, Lv H, Lu X, Guo X (2013) Blood pressure changes and chemical constituents of particulate air pollution: results from the healthy volunteer natural relocation (HVNR) study. Environ Health Perspect 121:66-72. https://doi.org/10.1289/ehp.1104812

51. Ye B, Ji X, Yang H, Yao X, Chan CK, Cadle SH, Chan T, Mulawa PA (2003) Concentration and chemical composition of PM 2.5 in Shanghai for a 1-year period. Atmos Environ 37:499-510. https://doi.org/10.1016/S1352-2310(02)00918-4

Figures

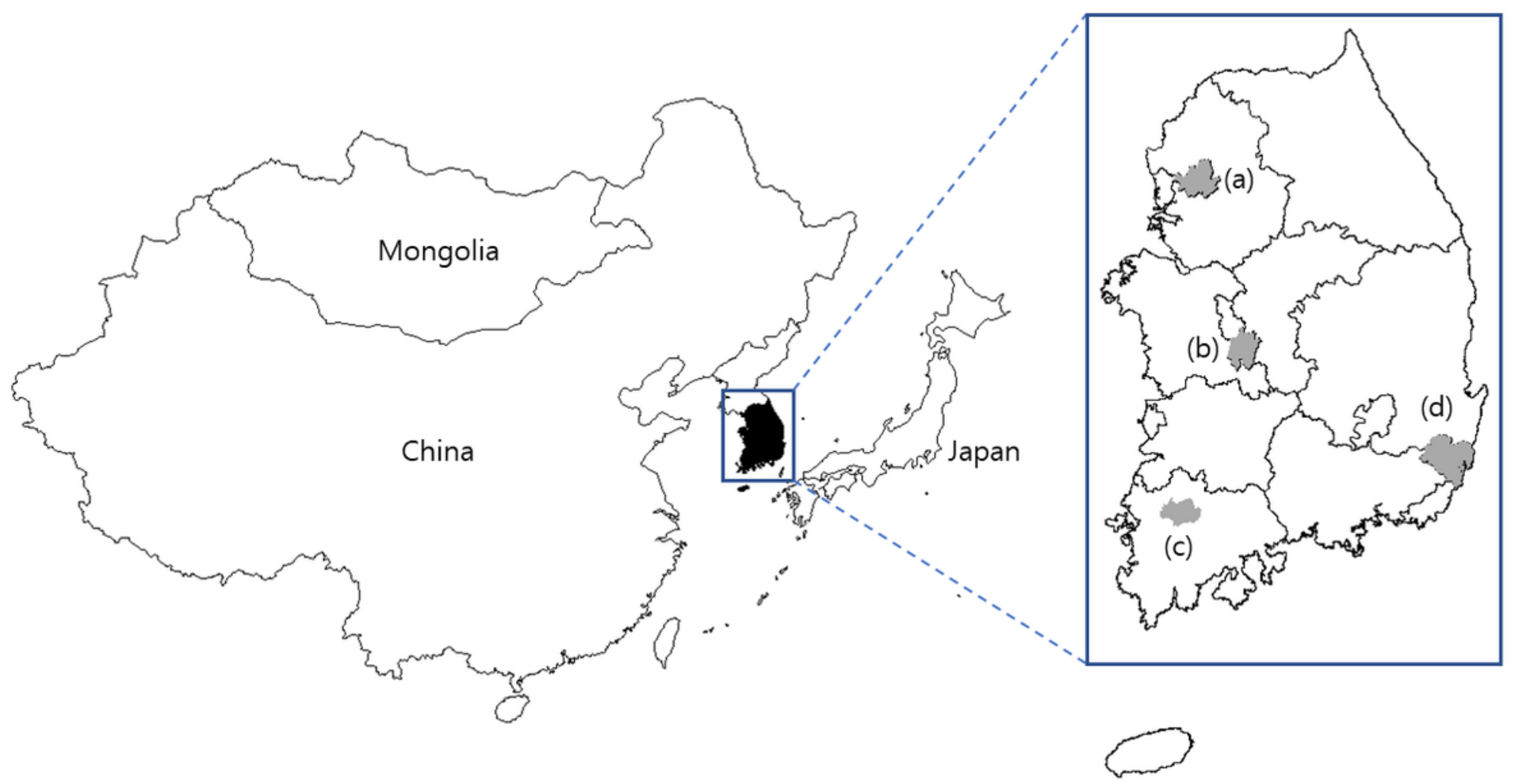

Figure 1

Geographical locations of the study cities; (a) SL, (b) DJ, (c) GJ and (d) US

\section{Supplementary Files}

This is a list of supplementary files associated with this preprint. Click to download.

- 5.SupplementaryinformationESPRfinal.docx 